\title{
Riemann Boundary Value Problem of Non-Normal Type on the Infinite Straight Line
}

\author{
Lixia Cao \\ Department of Information and Computing Sciences, Mathematics College, Northeast Petroleum University, Daqing, China \\ Email: caolixia98237@163.com
}

Received June 16, 2013; revised July 16, 2013; accepted July 25, 2013

Copyright (C) 2013 Lixia Cao. This is an open access article distributed under the Creative Commons Attribution License, which permits unrestricted use, distribution, and reproduction in any medium, provided the original work is properly cited.

\begin{abstract}
We consider a Riemann boundary value problem of non-normal type on the infinite straight line. By using the method of complex functions, we investigate the method for solving this Riemann boundary value problem of non-normal type and give the general solutions and the solvable conditions for it.
\end{abstract}

Keywords: Non-Normal Type; Riemann Boundary Value Problem; The Infinite Straight Line

\section{Introduction}

Various kinds of Riemann boundary value problems (BVPs) for analytic functions on closed curves or on open arc, doubly periodic Riemann BVPs, doubly quasi-periodic Riemann BVPs, and BVPs for polyanalytic functions have been widely investigated in [1-8]. The main approach is to use the decomposition of polyanalytic functions and their generalization to transform the boundary value problems to their corresponding boundary value problems for analytic functions. Recently, inverse Riemann BVPs for generalized analytic functions or bianalytic functions have been investigated in [9-12].

In this paper, we consider a kind of Riemann BVP of non-normal type on the infinite straight line and discuss the solvable conditions and the general solution for it.

\section{A Riemann Boundary Value Problem of Non-Normal Type on the Infinite Straight Line}

Let $X$ be the real axis oriented in the positive direction. And let $Z^{+}, Z^{-}$denote the upper half-plane and the lower half-plane cut by $X$. Our objective is to find a sectioally holomorphic function $\Phi(z)$ satisfying the following boundary condition

$$
\Phi^{+}(x)=\frac{\Pi_{1}(x)}{\Pi_{2}(x)} G(x) \Phi^{-}(x)+g(x), x \in X
$$

where the two given functions $G(x), g(x) \in \hat{H}(X)$ and $G(x) \neq 0$ on $X$ with $G(\infty)$ existing and $G(\infty) \neq 0$ (clearly $g(\infty)$ exists), and

$$
\Pi_{1}(x)=\prod_{j=1}^{m}\left(x-\alpha_{j}\right)^{\lambda_{j}}, \Pi_{2}(x)=\prod_{l=1}^{n}\left(x-\beta_{l}\right)^{\mu_{l}},
$$

with

$$
\alpha_{j}, \beta_{l}\left(\alpha_{j} \neq \beta_{l}\right)(j=1, \cdots, m ; l=1, \cdots, n)
$$

being on $X$ and $\lambda_{j}, \mu_{l} \in \mathbb{N}$. And the integer

$$
\kappa=\operatorname{Ind}_{X} G(x)=\frac{1}{2 \pi}[\arg G(x)]_{X}
$$

is the index of problem (1).

Write

$$
\lambda=\sum_{j=1}^{m} \lambda_{j}, \mu=\sum_{l=1}^{n} \mu_{l} .
$$

Without loss of generality, we can consider problem (1) in class $R_{0}$, that is, the two limits $\Phi^{+}(\infty)$ and $\Phi^{-}(\infty)$ exist as $z\left(\in \bar{Z}^{+}\right.$or $\left.\bar{Z}^{-}\right) \rightarrow \infty$. Clearly, here we have $\Phi^{ \pm}(+\infty)=\Phi^{ \pm}(-\infty)$.

\section{Homogeneous Problem}

The homogeneous problem of (1) is as follows

$$
\Phi^{+}(x)=\frac{\Pi_{1}(x)}{\Pi_{2}(x)} G(x) \Phi^{-}(x), \quad x \in X .
$$

It is found that $\lambda=\mu$ is required for solving problem (2) in class $R_{0}$. Here we suppose that $\lambda=\mu$. Let

$$
G_{0}(x)=\left(\frac{x+i}{x-i}\right)^{\kappa} G(x) \text {, }
$$


then $G_{0}(x) \in \hat{H}$ and $G_{0}(x) \neq 0$ with

$$
G_{0}(\infty)=G(\infty) \neq 0,
$$

and

$$
\operatorname{Ind}_{X} G_{0}(x)=\frac{1}{2 \pi}\left[\arg G_{0}(x)\right]_{X}=0 .
$$

Write

$$
\Gamma(z)=\frac{1}{2 \pi i} \int_{-\infty}^{+\infty} \frac{\log G_{0}(x)}{x-z} \mathrm{~d} x .
$$

Since $\operatorname{Ind}_{X} G_{0}(x)=0$, by taking logarithm of $G_{0}(x)$ for some branch we obtain a single-valued function $\log G_{0}(x)$ with $\log G_{0}(x) \in \hat{H}$, hence $\Gamma^{ \pm}(x)$ exists with $\Gamma^{ \pm}(x) \in \hat{H}$ and $\Gamma( \pm \infty)=0$. And by simple calculation we see that $\mathrm{e}^{\Gamma(z)}$ is sectionally holomorphic.

Write

$$
X(z)= \begin{cases}(z+i)^{-\kappa} \mathrm{e}^{\Gamma(z)}, & z \in Z^{+} ; \\ (z-i)^{-\kappa} \mathrm{e}^{\Gamma(z)}, & z \in Z^{-},\end{cases}
$$

then $X^{+}(x)=G_{0}(x) X^{-}(x)$. Substituting this into (2) gives

$$
\frac{\Pi_{2}(x) \Phi^{+}(x)}{X^{+}(x)}=\frac{\Pi_{1}(x) \Phi^{-}(x)}{X^{-}(x)} .
$$

If we write

$$
\Omega(z)= \begin{cases}\frac{\Pi_{2}(z) \Phi(z)}{X(z)}, & z \in Z^{+} ; \\ \frac{\Pi_{1}(z) \Phi(z)}{X(z)}, & z \in Z^{-},\end{cases}
$$

then we get $\Omega^{+}(x)=\Omega^{-}(x)$. Thus $\Omega(z)$ is analytic on the whole complex plane and has at most $\kappa+\lambda$ order at $\infty$. From [5], we know that $\Omega(z)$ must be an arbitrary polynomial $P_{\kappa+\lambda}(z)$ of degree $\kappa+\lambda$ with $P_{\kappa+\lambda}(z) \equiv 0$ if $\kappa+\lambda<0$. Therefore, the homogeneous problem (2) has general solution in class $R_{0}$ as follows

$$
\Phi(z)= \begin{cases}\frac{X(z) P_{\kappa+\lambda}(z)}{\Pi_{2}(z)}, & z \in Z^{+} ; \\ \frac{X(z) P_{\kappa+\lambda}(z)}{\Pi_{1}(z)}, & z \in Z^{-} .\end{cases}
$$

Considering the requirements that $\Phi^{+}(x)$ and $\Phi^{-}(x)$ are bounded at

$$
\alpha_{j}, \beta_{l}(j=1, \cdots, m ; l=1, \cdots, n),
$$

we can let

$$
P_{\kappa+\lambda}(z)=\Pi_{1}(z) \Pi_{2}(z) P_{\kappa-\lambda}(z),
$$

where $P_{\kappa-\lambda}(z)$ is an arbitrary polynomial of degree $\kappa-\lambda$ with $P_{\kappa-\lambda}(z) \equiv 0$ if $\kappa^{-\lambda}<0$. Now we get the general solution in class $R_{0}$ for the homogeneous problem (2) as follows

$$
\Phi(z)= \begin{cases}\Pi_{1}(z) X(z) P_{\kappa-\lambda}(z), & z \in Z^{+} ; \\ \Pi_{2}(z) X(z) P_{\kappa-\lambda}(z), & z \in Z^{-} .\end{cases}
$$

Thus we get the following results.

Theorem 3.1. For the homogeneous problem (2) in class $R_{0}$, the following two cases arise.

1) When $\kappa \geq \lambda$, it is always solvable and its general solution is given by (8), where $P_{\kappa-\lambda}(z)$ is an arbitrary polynomial of degree $\kappa-\lambda$.

2) When $\kappa<\lambda$, it only has zero-solution.

\section{Nonhomogeneous Problem}

For nonhomogeneous problem (1), the key is to find out the special solution.

Similar to the case in homogeneous problem (2), the canonical function $X(z)$ is given by (6) but with

$$
\Gamma(z)=\frac{1}{2 \pi i} \int_{-\infty}^{+\infty} \frac{\log G_{0}(x)}{x-z} \mathrm{~d} x(z \notin X)
$$

satisfying

$$
X^{+}(x)=G(x) X^{-}(x) .
$$

By this, problem (1) can be rewritten as

$$
\Pi_{2}(x) \frac{\Phi^{+}(x)}{X^{+}(x)}=\Pi_{1}(x) \frac{\Phi^{-}(x)}{X^{-}(x)}+\Pi_{2}(x) \frac{g(x)}{X^{+}(x)}, x \in X .
$$

We note that Plemelj formula can not be used directly here, because that when $\kappa>0$

$$
\lim _{x \rightarrow \infty} \frac{g(x)}{X^{+}(x)}=\lim _{x \rightarrow \infty}(x+i)^{\kappa} \mathrm{e}^{-\Gamma^{+}(x)} g(x)=\infty
$$

is not a finite constant, and so $\frac{g(x)}{X^{+}(x)} \notin \hat{H}$ (unless $\kappa \leq 0$ ). For a unified treatment, regardless of the value of $\kappa$, we always let

$$
Y(z)=(z+i)^{\kappa} X(z)= \begin{cases}\mathrm{e}^{\Gamma(z)}, & z \in Z^{+} ; \\ \left(\frac{z+i}{z-i}\right)^{\kappa} \mathrm{e}^{\Gamma(z)}, & z \in Z^{-} .\end{cases}
$$

Multiplying $(x+i)^{-\kappa}$ to the two sides of (9) gives

$$
\frac{\Pi_{2}(x) \Phi^{+}(x)}{Y^{+}(x)}=\frac{\Pi_{1}(x) \Phi^{-}(x)}{Y^{-}(x)}+\frac{\Pi_{2}(x) g(x)}{Y^{+}(x)} .
$$


We know that $\frac{g(x)}{Y^{+}(x)} \in \hat{H}$ and so that

$$
\frac{\Pi_{2}(x) g(x)}{Y^{+}(x)} \in \hat{H} .
$$

If we let

$$
\Psi(z)=\frac{1}{2 \pi i} \int_{-\infty}^{+\infty} \Pi_{2}(\tau) \frac{g(\tau)}{Y^{+}(\tau)} \frac{\mathrm{d} \tau}{\tau-z}, z \notin X,
$$

then we get

$$
\Psi^{+}(x)-\Psi^{-}(x)=\Pi_{2}(x) \frac{g(x)}{Y^{+}(x)}, \quad x \in X,
$$

and

$$
\begin{aligned}
& \frac{\Pi_{2}(x) \Phi^{+}(x)}{Y^{+}(x)}-\Psi^{+}(x) \\
= & \frac{\Pi_{1}(x) \Phi^{-}(x)}{Y^{-}(x)}-\Psi^{-}(x), x \in X,
\end{aligned}
$$

with $\Psi(\infty)=0$. Similar to the reasoning for (7) for problem (2), we know that if problem (1) has solution in class $R_{0}$, then can easily write out the form. But for problem (11), the function

$$
F(z)= \begin{cases}\frac{\Pi_{2}(z) \Phi(z)}{Y(z)}-\Psi(z), & z \in Z^{+}, \\ \frac{\Pi_{1}(z) \Phi(z)}{Y(z)}-\Psi(z), & z \in Z^{-}\end{cases}
$$

is analytic everywhere except at the possible unique pole $z=-i$, therefore the following two cases arise.

Case 1. $\kappa \geq 0$.

When $\kappa \geq 0, F(z)$ has a pole of order $\kappa$ at $z=-i$. To eliminate the singularity, we multiply $(z+i)^{\kappa}$ to $F(z)$ and get a polynomial of degree $\kappa+\lambda:$

$$
(z+i)^{\kappa} F(z)=P_{\kappa+\lambda}(z) .
$$

Therefore

$$
\Phi(z)= \begin{cases}\frac{X(z) P_{\kappa+\lambda}(z)+Y(z) \Psi(z)}{\Pi_{2}(z)}, & z \in Z^{+} ; \\ \frac{X(z) P_{\kappa+\lambda}(z)+Y(z) \Psi(z)}{\Pi_{1}(z)}, & z \in Z^{-}\end{cases}
$$

is actually the general solution for problem (1) in class $R_{0}$, where $(z+i)^{-\kappa} Y(z)=X(z)$. For convenience, we deform the function $\Phi(z)$ given by (12) into

$$
\Phi(z)=\left\{\begin{array}{l}
\frac{(z+i)^{-\kappa} \mathrm{e}^{\Gamma(z)}\left[P_{\kappa+\lambda}(z)+(z+i)^{\kappa} \Psi(z)\right]}{\Pi_{2}(z)}, z \in Z^{+} ; \\
\frac{(z-i)^{-\kappa} \mathrm{e}^{\Gamma(z)}\left[P_{\kappa+\lambda}(z)+(z+i)^{\kappa} \Psi(z)\right]}{\Pi_{1}(z)}, z \in Z^{-} .
\end{array}\right.
$$

Considering the requirements that $\Phi^{ \pm}(x)$ are bounded on $X$, and the fact that

$$
\begin{aligned}
\Phi^{+}(z) & =\frac{(z+i)^{-\kappa} e^{\Gamma(z)}\left[P_{\kappa+\lambda}(z)+(z+i)^{\kappa} \Psi(z)\right]}{\Pi_{2}(z)} \\
& =\frac{(z+i)^{-\kappa} e^{\Gamma(z)} F^{+}(z)}{\Pi_{2}(z)}
\end{aligned}
$$

with $F^{+}(z)=P_{\kappa+\lambda}(z)+(z+i)^{\kappa} \Psi(z)$, the smallest degree of $\left(t-\beta_{l}\right)$ in the numerator of the above formula should be $\mu_{l}$, we suppose that

$$
\begin{aligned}
F(t)= & a_{-n}\left(t-\beta_{l}\right)^{\mu_{l}+n}+\cdots+a_{0}\left(t-\beta_{l}\right)^{\mu_{l}} \\
& +a_{1}\left(t-\beta_{l}\right)^{\mu_{l}-1}+\cdots+a_{l}\left(t-\beta_{l}\right)+a_{l+1},
\end{aligned}
$$

then when $t \rightarrow \beta_{l}$, we have

$$
\begin{aligned}
& F(t) \rightarrow a_{l+1}, F^{\prime}(t) \rightarrow a_{l}, F^{\prime \prime}(t) \rightarrow 2 a_{l-1}, \cdots, \\
& F^{\left(\mu_{l}-1\right)}(t) \rightarrow a_{2}\left(\mu_{l}-1\right) !
\end{aligned}
$$

and the smallest degree of $\left(t-\beta_{l}\right)$ in numerator is $\mu_{l}$ (i.e. $\Phi^{+}(x)$ is bounded on $X$ ) only when

$$
F(t)=F^{\prime}(t)=F^{\prime \prime}(t)=\cdots=F^{\left(\mu_{l}-1\right)}(t)=0,
$$

that is,

$$
\begin{gathered}
P_{\kappa+\lambda}^{(s)}\left(\beta_{l}\right)+\left.\left[(z+i)^{\kappa} \Psi(z)\right]^{+(s)}\right|_{z=\beta_{l}}=0, \\
s=0,1, \cdots, \mu_{l}-1 ; l=1,2, \cdots, n .
\end{gathered}
$$

In a similar way, we know that $\Phi^{-}(x)$ is bounded on $X$ only when

$$
\begin{gathered}
P_{\kappa+\lambda}^{(r)}\left(\alpha_{j}\right)+\left.\left[(z+i)^{\kappa} \Psi(z)\right]^{-(r)}\right|_{z=\alpha_{j}}=0, \\
r=0,1, \cdots, \lambda_{j}-1 ; \quad j=1,2, \cdots, m
\end{gathered}
$$

are satisfied.

Hence, we get the results that $\Phi^{ \pm}(x)$ are bounded on $X$ only when the conditions (14) and (15) are all satisfied. While it is troublesome to solve the system composed by (14) and (15) for the coefficients of $P_{\kappa+\lambda}(z)$.

Here we aim to determine the coefficients by using Hermite interpolation polynomial.

Firstly, we make the polynomial $Q_{\rho}(z)$ of degree $\rho=\lambda+\mu-1=2 \lambda-1 \quad(\lambda=\mu)$ such that 


$$
\begin{gathered}
Q_{\rho}^{(r)}\left(\alpha_{j}\right)=\left.\left[(z+i)^{\kappa} \Psi(z)\right]^{-(r)}\right|_{z=\alpha_{j}}, r=0,1, \cdots, \lambda_{j}-1 ; j=1,2, \cdots, m, \\
Q_{\rho}^{(s)}\left(\beta_{l}\right)=\left.\left[(z+i)^{\kappa} \Psi(z)\right]^{+(s)}\right|_{z=\beta_{l}}, \quad s=0,1, \cdots, \mu_{l}-1 ; \quad l=1,2, \cdots, n .
\end{gathered}
$$

The polynomial $Q_{\rho}(z)$ exists uniguely from [5]. Let

$$
\Phi_{0}(z)= \begin{cases}\frac{(z+i)^{-\kappa} \mathrm{e}^{\Gamma(z)}\left[(z+i)^{\kappa} \Psi(z)-Q_{\rho}(z)\right]}{\Pi_{2}(z)}, & z \in Z^{+} ; \\ \frac{(z-i)^{-\kappa} \mathrm{e}^{\Gamma(z)}\left[(z+i)^{\kappa} \Psi(z)-Q_{\rho}(z)\right]}{\Pi_{1}(z)}, & z \in Z^{-} .\end{cases}
$$

We can see from (16) that $\Phi_{0}^{ \pm}(x)$ are continuous on $X$, and through simple verification that $\Phi_{0}^{ \pm}(x)$ satisfy the condition in problem (1), and that the order of $\Phi_{0}(z)$ at $\infty$ is $-\kappa+\rho-\lambda=\lambda-\kappa-1$.

Now we aim to make $\Phi_{0}(z)$ belong to $R_{0}$ by adding restricted conditions.

a) If $\lambda-\kappa-1 \leq 0$, that is, $\kappa \geq \lambda-1, \Phi_{0}(z)$ exactly belongs to $R_{0}$ and is justly the solution for homogeneous problem of (2), and also a particular solution for nonhomogeneous problem (1) in $R_{0}$. Combining with the general solution (8) of homogeneous problem (2), we known that when $\kappa \geq \lambda-1$, the general solution of problem (1) in $R_{0}$ is

$$
\Phi(z)= \begin{cases}\frac{(z+i)^{-\kappa} \mathrm{e}^{\Gamma(z)}\left[(z+i)^{\kappa} \Psi(z)-Q_{\rho}(z)\right]}{\Pi_{2}(z)}+\Pi_{1}(z) X(z) P_{\kappa-\lambda}(z), & z \in Z^{+} ; \\ \frac{(z-i)^{-\kappa} \mathrm{e}^{\Gamma(z)}\left[(z+i)^{\kappa} \Psi(z)-Q_{\rho}(z)\right]}{\Pi_{1}(z)}+\Pi_{2}(z) X(z) P_{\kappa-\lambda}(z), & z \in Z^{-},\end{cases}
$$

where $P_{\kappa-\lambda}(z)=0$ when $\kappa=\lambda-1$.

b) If $\lambda-\kappa-1>0$, that is, $\kappa<\lambda-1$, since the homogeneous problem (2) corresponding to (1) only has zero-solution in $R_{0}$, the existence of a solution $\Phi_{0}(z)$ for nonhomogeneous problem (1) in $R_{0}$ implies the uniqueness. Because $\Phi_{0}(z)$ has generally singularity of order $\lambda-\kappa-1$ at $\infty, \Phi_{0}(z)$ belongs to $R_{0}$ if and only if $\lambda-\kappa-1$ conditions on $g(x)$ or on $Q_{\rho}(z)$ are satisfied.

By rewriting $\Phi_{0}(z)$ as

$$
\Phi_{0}(z)= \begin{cases}\frac{(z+i)^{-\kappa} \mathrm{e}^{\Gamma(z)}\left[(z+i)^{\kappa} \Psi(z)-Q_{\rho}(z)\right]}{\Pi_{2}(z)}=\frac{\mathrm{e}^{\Gamma(z)} \Psi(z)}{\Pi_{2}(z)}-\frac{\mathrm{e}^{\Gamma(z)} Q_{\rho}(z)}{(z+i)^{\kappa} \Pi_{2}(z)}, & z \in Z^{+} ; \\ \frac{(z-i)^{-\kappa} \mathrm{e}^{\Gamma(z)}\left[(z+i)^{\kappa} \Psi(z)-Q_{\rho}(z)\right]}{\Pi_{1}(z)}=\frac{\mathrm{e}^{\Gamma(z)}(z+i)^{\kappa} \Psi(z)}{(z-i)^{\kappa} \Pi_{1}(z)}-\frac{\mathrm{e}^{\Gamma(z)} Q_{\rho}(z)}{(z-i)^{\kappa} \Pi_{1}(z)}, \quad z \in Z^{-} .\end{cases}
$$

Here we write

$$
Q_{\rho}(z)=A_{0} z^{\rho}+A_{1} z^{\rho-1}+\cdots+A_{\rho}(\rho=\lambda+\mu-1=2 \lambda-1) .
$$

Since the order of the denominator in $\Phi_{0}(z)$ is $\kappa+\lambda(\geq 1) \geq-1 \quad$ (is always true), it is enough that $Q_{\rho}(z)$ is at most a polynomial of degree $\kappa+\lambda$, that is,

$$
A_{0}=A_{1}=\cdots=A_{\lambda-\kappa-2}=0 \text {. }
$$

Therefore, only when condition (19) is satisfied, (16) is actually the general solution for nonhomogenous problem (1), now the homogeneous problem (2) only has zero-solution.

Case 2. $\kappa<0$.

When $\kappa<0, F(z)$ is analytic on the whole complex plane and has $\lambda$ order at $\infty$, that is,

$F(z)=P_{\lambda}(z)$, and now the homogeneous problem (2) only has zero-solution. Therefore the general solution for nonhomogenous problem (1) in $R_{0}$ is given by

$$
\Phi(z)=\left\{\begin{array}{ll}
\frac{\left[P_{\lambda}(z)+\Psi(z)\right] Y(z)}{\Pi_{2}(z)} \\
\frac{\left[P_{\lambda}(z)+\Psi(z)\right] Y(z)}{\Pi_{1}(z)}
\end{array}= \begin{cases}\frac{\left[P_{\lambda}(z)+\Psi(z)\right]}{\Pi_{2}(z)} \mathrm{e}^{\Gamma(z)}, & z \in Z^{+} ; \\
\frac{\left[P_{\lambda}(z)+\Psi(z)\right]}{\Pi_{1}(z)}\left(\frac{z+i}{z-i}\right)^{\kappa} \mathrm{e}^{\Gamma(z)}, & z \in Z^{-} .\end{cases}\right.
$$


If we make the Hermite interpolation polynomial of degree $\lambda-1$, the fact that $\lambda-1<\lambda$ (the order of the denominator) implies that $\Phi(z)$ is unbounded on $X$, which contradicts with the hypothesis that $\Phi(z)$ is bounded on $X$. So it is infeasible to make the Hermite interpolation polynomial for this.

However, we have the following effective treatment for this.

a) Under this situation, $\Phi(z)$ may have the unique pole at $z=-i$. In order to eliminate the pole, we should put the following restrictions for it:

if $\kappa+\lambda \geq 0$, we only need

$$
P_{\lambda}^{(j)}(-i)+\Psi^{(j)}(-i)=0, j=0,1,2, \cdots,-\kappa-1 ;
$$

if $\kappa+\lambda=-1$, we only need to put

$$
P_{\lambda}(-i)=-\Psi(-i) ;
$$

if $\kappa+\lambda<-1$, apart from (22), the restrictions

$$
\Psi^{(j)}(-i)=0, j=1,2, \cdots,-\kappa-1,
$$

or

$$
\int_{-\infty}^{+\infty} \frac{\Pi_{2}(x) g(x)}{Y^{+}(x)(x+i)^{j+1}} \mathrm{~d} x=0, j=1,2, \cdots,-\kappa-1
$$

are necessary.

b) Considering the boundedness of $\Phi(z)$ on $X$, the following restrictions are also necessary:

$$
\begin{gathered}
P_{\lambda}^{(s)}\left(\alpha_{j}\right)+\Psi^{-(s)}\left(\alpha_{j}\right)=0, r=0,1, \cdots, \lambda_{j}-1 ; j=1,2, \cdots, m \\
P_{\lambda}^{(s)}\left(\beta_{l}\right)+\Psi^{+(s)}\left(\beta_{l}\right)=0, s=0,1, \cdots, \mu_{l}-1 ; l=1,2, \cdots, n .
\end{gathered}
$$

Thus we get the following results.

Theorem 4.1. For the nonhomogeneous problem (1) in class $R_{0}$, the following two cases arise.

1) $\kappa \geq 0$. If $\kappa \geq \lambda-1$, problem (1) is always solvable and its general solution is given by (17); and if $\kappa<\lambda-1$, if and only if $Q_{\rho}(z)$ satisfies condition (19), problem (1) has unique solution, given by (16).

2) When $\kappa<0$, it has unique solution in form (20) when the restrictions (21) or (22) or (22)-(23), and (24)(25) are satisfied.

Anyway, the degree of freedom of solution for nonhomogeneous problem (1) is $\kappa-\lambda+1$.

\section{REFERENCES}

[1] M. B. Balk, "Polyanalytic Functions," Akademie Verlag, Berlin, 1991.

[2] H. Begehr and A. Kumar, "Boundary Value Problems for the Inhomogeneous Polyanalytic Equation I," Analysis: International Mathematical Journal of Analysis and Its Application, Vol. 25, No. 1, 2005, pp. 55-71.

[3] D. Jinyuan and W. Yufeng, "On Boundary Value Problems of Polyanalytic Functions on the Real Axis," Complex Variables, Vol. 48, No. 6, 2003, pp. 527-542. doi:10.1080/0278107031000103412

[4] B. F. Fatulaev, "The Main Haseman Type Boundary Value Problem for Metaanalytic Function in the Case of Circular Domains," Mathematical Modelling and Analysis, Vol. 6, No. 1, 2001, pp. 68-76.

[5] J. K. Lu, "Boundary Value Problems for Analytic Functions," World Scientific, Singapore City, 1993.

[6] A. S. Mshimba, "A Mixed Boundary Value Problem for Polyanalytic Function of Order $n$ in the Sobolev Space Wn, p(D)," Complex Variables, Vol. 47, No. 12, 2002, pp. 278-1077.

[7] N. I. Muskhelishvili, "Singular Integral Equations," World Scientific, Singapore City, 1993.

[8] W. Yufeng and D. Jinyuan, "Hilbert Boundary Value Problems of Polyanalytic Functions on the Unit Circumference," Complex Variables and Elliptic Equations, Vol. 51, No. 8-11, 2006, pp. 923-943. doi:10.1080/17476930600667692

[9] L. Xing, "A Class of Periodic Riemann Boundary Value Inverse Problems," Proceedings of the Second Asian Mathematical Conference, Nakhon Ratchasima, 17-20 October 1995, pp. 397-400.

[10] M. H. Wang, "Inverse Riemann Boundary Value Problems for Generalized Analytic Functions," Journal of Ningxia University of Natural Resources and Life Sciences Education, Vol. 27, No. 1, 2006, pp. 18-24.

[11] X. Q. Wen and M. Z. Li, "A Class of Inverse Riemann Boundary Value Problems for Generalized Holomorphic Functions," Journal of Mathematical, Vol. 24, No. 4, 2004, pp. 457-464.

[12] L. X. Cao, P.-R. Li and P. Sun, "The Hilbert Boundary Value Problem With Parametric Unknown Function on Upper Half-Plane," Mathematics in Practice and Theory, Vol. 42, No. 2, 2012, pp. 189-194. 